\title{
Psychische Belastung erhöht Schlaganfallrisiko bei Älteren
}

punktwert für die psychische Belastung errechnet, wobei höhere Punktwerte eine stärkere Belastung anzeigen.

Während der durchschnittlich sechs Jahre betragenden Nachbeobachtungszeit traten 151 tödliche und 452 nicht tödliche Schlaganfälle auf. Im Vergleich zu Personen mit geringer psychischer Belastung (unterstes Viertel der Punktwerte) hatten Personen mit der höchs- ten psychischen Belastung (oberstes Viertel) ein fast dreifach erhöhtes Risiko für einen tödlichen Schlaganfall (Hazard Ratio: 2,97) und ein um 54\% erhöhtes Risiko für einen nicht tödlichen Schlaganfall (Hazard Ratio 1,54).

\footnotetext{
- Henderson et al.

Psychosocial distress and stroke risk in older adults. Stroke 2013; 44: 367-372
}

\section{Kommentar}

Während vorangehende Studien mehrheitlich den Zusammenhang zwischen Depression und Schlaganfall untersucht hatten, zeigt diese Studie, dass auch für eine breitere Definition von psychischer Belastung ein Einfluss auf das Schlaganfallrisiko besteht. Dabei fand sich eine klare Dosis-WirkungsBeziehung mit kontinuierlich ansteigendem Risiko bei zunehmender psychischer Belastung.

Ursächlich könnten neuroendokrine, neurovegetative und inflammatorische Prozesse eine Rolle spielen. Darüber hinaus ist zu berücksichtigen, dass psychische Belastung oftmals mit ungesunder Lebensweise durch Bewegungsmangel, Rauchen und ungesunder Ernährung einhergeht. Psychische Belastung ist ein potenziell modifizierbarer Risikofaktor. In zukünftigen Studien sollte daher vor allem untersucht werden, ob hierauf abzielende Interventionen (z. B. mit gezieltem Screening, Informationen für Betroffene und Angehörige, medizinischer oder psychotherapeutischer Behandlung) bei älteren Menschen in der Schlaganfallprävention wirksam sind.

M. BusCH =

\section{Ramipril verlängert Gehstrecke bei PAVK}

\section{Patienten mit Claudicatio intermit- tens haben nach einer halbjährigen Behandlung mit Ramipril einen deut- lichen Anstieg der schmerzfreien Gehstrecke und der maximalen Geh- dauer auf dem Laufband.}

- Ramipril wirkt sich bei Patienten mit koronarer Herzerkrankung nachweislich günstig auf Morbidität und Mortalität aus, bei der PAVK gab es bislang nur einige kleine Pilotstudien mit allerdings vielversprechenden Ergebnissen. Nun wurde eine randomisierte und placebokontrollierte Studie an 212 Patienten im Durchschnittsalter von 66 Jahren mit PAVK vorgelegt, die über 24 Wochen hinweg entweder $10 \mathrm{mg}$ Ramipril oder Placebo erhielten.
Nach sechs Monaten stellte man in der Ramiprilgruppe eine hoch signifikante Zunahme der schmerzfreien Gehstrecke auf dem Laufband um $75 \mathrm{~s}$ und der Dauer der maximalen Gehgeschwindigkeit um $255 \mathrm{~s}$ fest.

Auch der Knöchel-Oberarm-Index ABI sowohl in Ruhe als auch nach Belastung nahm gering, aber doch signifikant zu. Das Ausmaß des Effektes war bei Patienten mit einer Gefäßerkrankung im Femoropoplitealbereich größer als bei einer aortoiliakalen Angiopathie.

\footnotetext{
- A. A. Ahimastos et al.

Effect of ramipril on walking times and quality of life among patients with peripheral artery disease and intermittent claudication. JAMA 2013; 309: 453-460.
}

\section{Kommentar}

Über die Wirkmechanismen einer ACEHemmung zur Linderung ischämischer Symptome besteht Unklarheit. Interessanterweise stellte man in der vorliegenden Studie fest, dass der Blutfluss in offenen Gefäßen proximal der Stenose zunahm, nicht jedoch distal der Stenose. Dieser Befund spricht dafür, dass Ramipril offensichtlich keinen Effekt auf die Stenose selbst hat, sondern zu einer Dilatation oder sogar Neubildung von Kollateralgefäßen führt und somit die Durchblutungssituation distal der Stenose bessert. Man darf gespannt sein, ob der Effekt auch über die in der vorliegenden Studie untersuchten sechs Monate hinaus anhält.

H. S. FÜESSL = 